\title{
Tertulia literaria virtual como estrategia para la mejora de la convivencia escolar
}

\author{
Virtual literary gathering as a strategy to improve school coexistence
}

\author{
Gely Coraly Martinez-Ubillús « 1,a \\ https://orcid.org/OOOO-0002-5617-5681 \\ Gilberto Carrión-Barco 1,b \\ https://orcid.org/0000-0002-1104-6229
}

Recibido: 02-10-2020

Arbitrado por pares

Aceptado: 01-01-2021

\section{Citar como}

Martinez-Ubillús, G. C. y Carrión-Barco, G. (2020). Tertulia literaria virtual como estrategia para la mejora de la convivencia escolar. Desafíos, 12(1); 29-36. https://doi.org/10.37711/desafios.2021.12.1.268

\section{RESUMEN}

Objetivo. El objetivo principal del presente estudio es diseñar la estrategia de tertulia literaria virtual para mejorar la convivencia escolar de la Institución Educativa Mallaritos, respondiendo a una problemática de la población en estudio. Métodos. Para esta investigación se utilizó el diseño no experimental que se aplicó de manera transversal. Así mismo se aplicó un cuestionario con 22 ítems dividido en cuatro dimensiones: relaciones interpersonales, disciplina democrática, relación con el contexto y ciberconducta, aplicadas a una muestra de 43 estudiantes del cuarto grado de secundaria. La validez de los instrumentos se hizo bajo tres aspectos: validez de criterio, validez de constructo y validez de contenido determinada a través del juicio de expertos. Los cuales calificaron la pertinencia y relevancia de este. La confiabilidad se midió a través de Cronbach y Mc Donald en donde se obtuvo 0,952 en ambos resultados. Resultados. Los hallazgos fueron poco favorables en cuanto a la variable en estudio, se obtuvo el nivel bajo y medio de 30 \% y 42 \%, respectivamente, razón por la cual el objetivo propuesto. Conclusiones. Se concluye que es preciso aplicar una estrategia de tertulia literaria virtual que permita la mejora de la convivencia escolar en dicha institución por ser necesaria para los aprendizajes en un ambiente colaborativo.

Palabras clave: convivencia; tertulia; aprendizaje; estudiantes; lectura.

\section{ABSTRACT}

Objective. The main objective of this study is to design a virtual literary gathering strategy to improve school coexistence at the Mallaritos Educational Institution, responding to a problem of the population under study. Methods. A non-experimental design was used for this research, which was applied in a cross-sectional manner. A questionnaire with 22 items divided into four dimensions was also applied: interpersonal relationships, democratic discipline, relationship with the context and cyberbehavior, applied to a sample of 43 students in the fourth grade of high school. The validity of the instruments was assessed under three aspects: criterion validity, construct validity and content validity determined through the judgment of experts. These experts rated the pertinence and relevance of the instrument. Reliability was measured through Cronbach's and McDonald's test, where 0.952 was obtained in both results. Results. The findings were unfavorable in terms of the variable under study, the low and medium levels of $30 \%$ and $42 \%$, respectively, were obtained, which is why the proposed objective. Conclusions. It is concluded that it is necessary to apply a virtual literary gathering strategy that allows the improvement of school coexistence in this institution because it is necessary for learning in a collaborative environment.

Keywords: coexistence; social gathering; learning; students; reading.
\end{abstract}

\footnotetext{
Filiación y grado académico

1 Universidad César Vallejo, Piura, Perú.

a Magister en Educación con mención en Docencia y Gestión Educativa.

b Doctor en Ciencias de la Computación y Sistemas.
} 


\section{INTRODUCCIÓN}

A nivel internacional, la UNESCO dio a conocer en el 2019 un informe hecho en 144 países, en donde casi uno de cada tres estudiantes (32\%) ha sido intimidado por sus compañeros en la escuela, al menos una vez en el último mes, y una proporción similar se ha visto afectada por la violencia física, según la publicación. La intimidación física es la más frecuente en muchas regiones, con la excepción de Norteamérica y Europa, donde es más común la intimidación psicológica. El acoso sexual es el segundo tipo de acoso más común en muchas regiones. La violencia escolar y la intimidación afectan tanto a los alumnos como a las alumnas. El acoso físico es más común entre los niños, mientras que el psicológico es más frecuente entre las niñas. Además, aumentan también el acoso en línea y por teléfono móvil, dice el informe (UNESCO, 2019).

La mayoría de los países de América Latina han promovido políticas en relación con la convivencia escolar. Sin embargo, pueden distinguirse distintos énfasis en su conceptualización. Investigaciones en 18 países así lo demuestran, identificándose tres aspectos importantes: convivencia escolar democrática, seguridad ciudadana, y salud mental infanto-juvenil (Morales y López, 2019). Entre enero y noviembre del 2019 se reportaron 11934 casos de violencia escolar en colegios públicos y privados de todo el Perú, siendo el maltrato físico el que ocupó el primer lugar, según informó el Ministerio de Educación (Andina, 2019).

Por su parte, el Sistema Especializado en reporte de casos sobre Violencia Escolar (SíseVe) del Ministerio de Educación (MINEDU), sostiene que entre los meses de setiembre del 2013 y enero del 2019 , el 53 \% de casos (20 694) informados a nivel nacional perteneció a violencia entre pares, y el $47 \%$ (18 621) a agresiones por parte del personal de la institución educativa contra las y los estudiantes (Arrunátegui, 2020).

Según el MINEDU (2018), la convivencia escolar es un conjunto de relaciones humanas que toman lugar en una institución educativa, se forman de manera unificada entre todos sus miembros y todos ellos son responsables de la misma. Así mismo, en el 2019, el $72 \%$ de las víctimas denuncian haber sido agredidas por Facebook, 40 \% por Whatsapp, $10 \%$ Instagram y 3 \% por Twitter (Redacción EC, 2019).

La tertulia literaria es una estrategia que se viene implementando a nivel del MINEDU (2016), fomentando el gusto por la lectura, para fomentar una cultura de paz y una convivencia armónica. Por lo mismo, es fundamental el conjunto de esfuerzos que se hagan para ello, tanto a nivel nacional, regional y local. En el marco de esta situación de emergencia nacional y puesto que los estudiantes trabajan de manera virtual, se tiene la necesidad de implementar las tertulias literarias de manera remota

La Institución Educativa Complejo Mallaritos se encuentra ubicada en el distrito de Marcavelica, provincia de Sullana. Su localización exacta es el Centro Poblado Mallaritos. Es una escuela en donde sus estudiantes provienen, en su mayoría de hogares disfuncionales. Esta situación descrita requiere encontrar respuesta a la interrogante de investigación, ¿de qué manera la estrategia de Tertulia Literaria Virtual mejora la convivencia escolar en la Institución Educativa Complejo Mallaritos?

Carozzo (2016) afirma que la convivencia es una construcción de muchos años en la escuela, y esto no quiere decir que tengamos una concepción correcta de la misma. Para la mayoría de los directivos, docentes y padres de familia la convivencia es sinónimo de disciplina, respeto y orden, siendo renuentes a la idea que la convivencia sea una real interacción entre toda la comunidad educativa.

Según sostienes Álvarez et al. (2012), las tertulias literarias son consideradas por la comunidad de aprendizaje como una actuación educativa de éxito; esto quiere decir que ha sido aplicada en otros lugares y ha tenido éxito; es así que hay infinidad de lecturas que originan tertulias literarias, desde las que corresponden a contenidos matemáticos hasta aquellas que abarcan contenidos deportivos y de entretenimiento. Sin embargo, una de las que mayor apogeo ha conseguido en las últimas décadas es la tertulia literaria conocida en las comunidades de aprendizaje dialógico. Los miembros y el moderador se sientan en círculo de tal manera que están al mismo nivel, sin ninguna función de poder.

Durante este periodo de aislamiento social obligatorio (cuarentena), la educación ha sido posible a través del uso del Internet y se ha continuado con el aprendizaje de los niños, niñas y adolescentes del Perú. Por lo tanto, se ve que los mismos usan con más frecuencia las tecnologías de la información y comunicación (TIC), a través de chats, aulas virtuales, videollamadas, transmisiones en vivo, aplicativos, ente otros; permitiendo así continuar el desarrollo de sus competencias (MINEDU, 2020).

Por tanto, el uso del Internet en este momento se ha hecho indispensable en la educación a distancia, siendo urgente que nos cuenta y prevenir los riegos que pueden surgir de este confinamiento. 
La estrategia de tertulia literaria virtual tiene como referente la tertulia literaria dialógica del proyecto comunidades de aprendizaje, desarrollada como una actuación educativa de éxito. De acuerdo con Álvarez et al. (2012), está orientada a promover el diálogo igualitario entre los participantes de esta y el poder romper cualquier barrera de poder la enriquece. Inclusive, el docente asume una función de moderador que no evalúa la misma, sino simplemente dirige la participación de sus miembros.

Como objetivo se buscó diseñar la estrategia de tertulia literaria virtual para mejorar la convivencia escolar de la Institución Educativa Mallaritos. Para lo cual se analizó tres conceptos importantes. Primero la convivencia escolar que en general, hace referencia a las relaciones interpersonales que se tienen en la escuela como parte de la pedagogía misma; destacando su valor regulador, formativo y preventivo haciendo que esta convivencia vaya más allá que sólo la disciplina escolar. Otro concepto que se debe analizar es el de clima escolar pues tiene mucho que ver con el clima organizacional dentro de un sistema laboral; pues, si bien es cierto que la escuela es una organización laboral donde diferentes personas realizan un trabajo, es así mismo una comunidad educativa que promueve el desarrollo integral de los y las estudiantes. Finalmente, la cultura escolar que tiene mucha relación con la convivencia y el clima escolar, pues cada escuela es única y diferente en cuanto a su comunidad educativa, también es diferente a cualquier otra organización. Incluso dentro de un mismo distrito dos escuelas no son las mismas. Por lo tanto, los tres conceptos van estrechamente relacionados (MINEDU, 2017).

\section{MÉTODOS}

\section{Tipo de estudio}

La investigación fue de tipo propositiva bajo un enfoque cuantitativo. Este enfoque se basa en obtener resultados numéricos para ser procesados por la estadística (Hernández eł al., 2014). Así mismo, se escogió un tipo de estudio descriptivo, el cual permitió caracterizar a cada una de las dimensiones relacionadas con la convivencia escolar.

El diseño fue no experimental, debido a que no existió manipulación ninguna de las variables en estudio: convivencia escolar y tertulia literaria virtual; es decir, se recogió la información tal cual se encontraba en la muestra tomada. Asimismo, es de corte transversal porque se realizó en un único momento (Rodríguez y Mendivelso, 2018).

\section{Variables de estudio}

Como variables se tiene a la variable dependiente: convivencia escolar; y a la variable independiente: tertulias literarias virtuales.

\section{Convivencia escolar}

La variable fue analizada mediante la aplicación de un cuestionario con 22 preguntas cerradas en escala de Likert, a los alumnos del nivel secundaria de la Institución Educativa Mallaritos.

\section{Tertulias literarias virtuales}

la variable estuvo conformada por un conjunto de actividades que consisten en reunir a los estudiantes de manera virtual para tratar un tema importante para ellos a fin de que mejore su convivencia escolar dentro de la Institución Educativa Mallaritos.

\section{Población y muestra}

La población estuvo conformada por 68 estudiantes de cuarto año de secundaria de la I. .E Complejo Educativo Mallaritos, divididos en tres secciones: A, B y C, cuyas características sociodemográficas comprenden las edades entre 15, y 16 años, de ambos sexos, pertenecientes a un nivel socioeconómico medio bajo, donde los padres de familia, en su mayoría, solo tienen estudios secundarios y en su mayoría y son agricultores. La muestra es no probabilística y estuvo conformada por 43 estudiantes de las secciones A y B, usando criterios de exclusión por conveniencia, siendo ésta dos secciones las que poseen mayor conectividad (Arias et al., 2016)

\section{Instrumentos de recolección de datos}

La técnica que se usó para la recolección de datos fue la encuesta y como instrumento el cuestionario (Casas et al., 2003). La encuesta se diseñó en base al modelo teórico propuesto por Yuni y Urbano (2014), teniendo en consideración 4 dimensiones que permiten realizar una medición sobre el nivel de convivencia escolar en estudiantes de nivel secundaria; mientras que la escala de Likert tuvo los siguientes valores: nunca (1), casi nunca (2), a veces (3), casi siempre (4) y siempre (5).

Así mismo se obtuvo el coeficiente omega, obteniendo 0,952; lo cual prueba que el instrumento es altamente confiable. Para considerar un valor aceptable de confiabilidad a través el coeficiente omega, éstos deben encontrarse entre $0.70 \mathrm{y}$, por consiguiente, los resultados obtenidos en el presente trabajo de investigación son plenamente confiables.

\section{Procedimientos de la recolección de datos}

Se utilizó el test de convivencia escolar de elaboración propia para estudiantes de cuarto año 
de educación secundaria, el cual se aplicó a 43 estudiantes de la Institución Educativa Malllaritos. El instrumento estuvo constituido por 22 ítems, con cinco opciones de respuesta múltiple: casi nunca, nunca, a veces, casi siempre y siempre; del cual los primeros seis (06) îtems midieron la dimensión "relaciones interpersonales" (D1); los siguientes ocho (08) îtems evaluaron la dimensión "disciplina democrática" (D2); los cuatro (04) siguientes midieron la dimensión "relación con su contexto" (D3); y los últimos cuatro (04) ítems midieron la "ciberconducta" (D4). El instrumento se aplicó a cada estudiante en un solo momento. Los resultados se trasladaron a una base de datos construida en Excel, la cual se utilizó posteriormente para el estudio descriptivo e inferencial de los datos en SPSS.

\section{Aspectos éticos.}

Se coordinó y solicitó la autorización de la directora de la I. E. Complejo Mallaritos, para la aplicación del instrumento. Luego, se informó a los estudiantes sobre los beneficios de participar en el estudio planteado y, también, sobre la confidencialidad de los resultados al recoger la información de forma anónima. Seguidamente, se aplicó el instrumento en la muestra; lo que permitió ser la base del estudio.

Entre los aspectos éticos, se consideraron el anonimato de los estudiantes integrantes de la muestra; es una investigación original que contó con la autorización de la directora, docentes, padres de familia y estudiantes de la Institución Educativa en estudio; no se juzgaron las respuestas que brindaran los estudiantes; se colocaron en las referencias a todos los autores que aportaron con la fundamentación teórica y científica en la presente investigación.

\section{Desarrollo de la intervención.}

El modelo se desarrollará mediante la aplicación de diez sesiones de tertulia literaria virtual. Para ello se eligió la obra clásica Las mil y una noches, versión digital en pdf para ser enviada de manera virtual a los estudiantes. Se elegirá la plataforma a usar, poniendo la más usada entre todos sus miembros. Semanalmente se hará una sesión de tertulia literaria virtual, la misma que tendrá como acuerdo, al final de la misma, las páginas que serán leídas en la próxima sesión. Así mismo, en cada reunión se hará un checklist que consistirá en criterios que se deberán de tener en cuenta al término de cada sesión, sin ser esta una evaluación.

En cada sesión de tertulia literaria virtual, el moderador hará una lista de turnos de participación, respetándola en todo momento. Del mismo modo, habrá intervenciones no contempladas en la lista, pero que surgirán de la participación de cada estudiante, acotando y reforzando la idea o párrafo leído. Cada participante deberá seleccionar el párrafo que más le ha gustado y, al mismo tiempo, lo leerá para todos y deberá contar por qué es significativo para él y de qué manera lo puede relacionar con alguna experiencia de su vida.

Esta práctica constante y semanal promoverá el diálogo en todo momento y generará, poco a poco, la participación de todos sus miembros, promoviendo una cultura de paz y respeto, aun de manera virtual, por ser el momento en el que se vive.

Siendo que el propósito de la estrategia de tertulia literaria virtual es la mejora de la convivencia escolar, fueron desarrollas las siguientes actividades:

\section{Desarrollo de Actividades}

1. Fase de sensibilización de toda la comunidad educativa, en donde se conocieron los objetivos a ser alcanzados y se comprometieron con su realización (responsable, acción y fecha).

2. La escuela abrió sus puertas a la comunidad (familiares, agentes comunitarios, vecinos, etc.) que quieran la mejora de la convivencia escolar.

3. Como la tertulia es una actuación de éxito que ya ha sido comprobada en otros escenarios, ahora se desarrollará de manera virtual.

4. Se elegirá el texto a trabajar, que deberá ser un clásico de la literatura, de acuerdo con el grado de los estudiantes. En este caso Las mil y una noches (antología de cuentos orientales).

5. En este contexto de educación remota en el que se vive, nació la iniciativa de hacer tertulias literarias con estudiantes de Cuarto grado de educación secundaria, pues estas son la muestra en cuestión de la investigación.

6. Se utilizaron diversos medios para aplicar esta tertulia literaria como plataformas de videoconferencia virtual (Zoom, Meet, Skype or WhatsApp).

7. La estrategia estuvo pensada para hacerla un bimestre o 2 meses, dividida en 10 sesiones.

De esta manera, al dialogar con los estudiantes sobre sus experiencias de vida en un ambiente de igualdad, se promoverá una sana convivencia de manera virtual, en estos tiempos en donde las redes sociales han incrementado considerablemente su uso.

\section{Análisis de datos}

Mediante pruebas estadísticas, se realizó el análisis de los datos procesados por medio del análisis descriptivo, el cual fue utilizado para estudiar y describir los datos recolectados de la muestra, presentados en forma de tablas y gráficos con las interpretaciones de los mismos; y finalmente, 
Tabla 1

Calificación de la variable dependiente: convivencia escolar

\begin{tabular}{lccc}
\hline \multirow{2}{*}{ Rango } & Calificación & \multicolumn{2}{c}{ VD:Convivencia Escolar } \\
\cline { 3 - 4 } & & $\mathrm{fi}$ & $\%$ \\
\hline $0-56$ & Bajo & 13 & 30 \\
$57-78$ & Medio & 18 & 42 \\
$79-100$ & Alto & 12 & 28 \\
\hline
\end{tabular}

se propone la estrategia de tertulia literaria virtual para la I. E. Complejo Mallaritos, 2020.

\section{RESULTADOS}

Para comenzar, se tenía que saber cómo estaba la convivencia escolar de manera virtual, pues ahora todo se maneja a través de grupos de WhatsApp. Para lo cual se aplicó una encuesta cuyos resultados fueron los siguientes:

La tabla 1 muestra que, en la Institución Educativa, los estudiantes del cuarto año de educación secundaria con respecto a la convivencia escolar se encuentran en un $28 \%$, en nivel alto, el $42 \%$ en un nivel bajo y el $30 \%$ en el nivel medio; con lo que se infiere que la variable dependiente Convivencia Escolar en la Institución Educativa se encuentra entre en un bajo nivel; lo que conduce a concluir que necesita mayor atención por parte de los directivos de la entidad
Tabla 2

Calificación la variable convivencia escolar por dimensiones

\begin{tabular}{lcccc}
\hline Calificación & \multicolumn{4}{c}{$\%$} \\
\cline { 2 - 5 } & $\begin{array}{c}\text { Relaciones } \\
\text { inter- } \\
\text { personales }\end{array}$ & $\begin{array}{c}\text { Disciplina } \\
\text { democrática }\end{array}$ & $\begin{array}{c}\text { Relación con } \\
\text { el contexto }\end{array}$ & Ciberconducta \\
\hline Bajo & 30 & 60 & 44 & 26 \\
Medio & 42 & 40 & 35 & 56 \\
Alto & 28 & 0 & 21 & 19 \\
\hline
\end{tabular}

A través de la tabla 2 se evidencia que la dimensión "relaciones interpersonales", es considerada por el 42 \% de los estudiantes en un nivel medio; por consiguiente, se necesita tomar conciencia de la realidad presentada. Con respecto a la dimensión "disciplina democrática" es calificada con un nivel bajo por el $60 \%$ de los estudiantes encuestados; lo cual requiere de elaborar acuerdos de convivencia participativa. Así mismo, la dimensión "relación con el contexto" se ubica en un nivel bajo por el $44 \%$ de los estudiantes; lo que conlleva a mirar atentamente a la comunidad educativa. Finalmente, la dimensión "ciberconducta" es considerada en un nivel medio por el 56 \% de los estudiantes de la Institución Educativa Complejo Mallaritos.

Al mismo tiempo, se presenta, la siguiente propuesta (ver figura 1), la cual interrelaciona los principios del aprendizaje dialógico con las dimensiones aplicadas en el cuestionario y los materiales a usar.

Propuesta de tertulias literarias virtuales
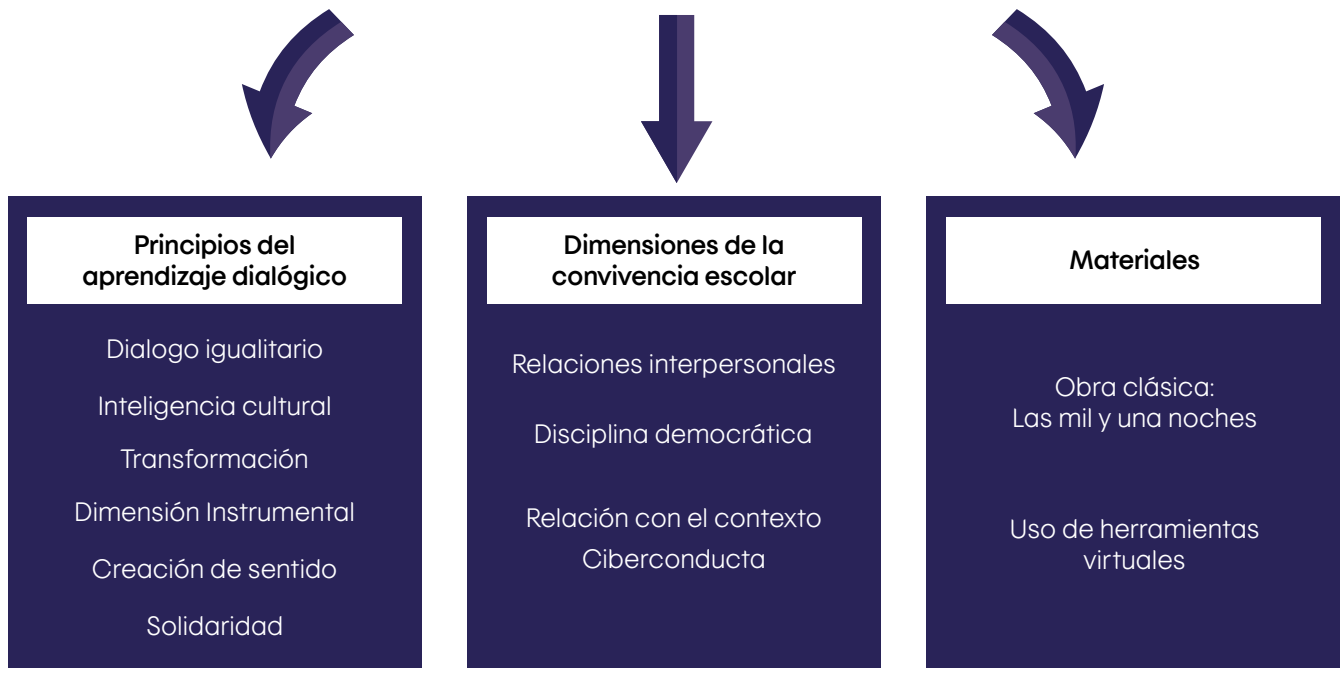

Figura 1. Propuesta de tertulias literarias 


\section{DISCUSIÓN}

A la luz de los resultados, poco favorables en cuanto a la convivencia escolar, se propone elaborar una estrategia de tertulia literaria virtual, cuyo objetivo es promover una convivencia democrática que permita que los estudiantes propongan sus normas de convivencia y trabajen colaborativamente para lograr cambios positivos en la misma.

Con el fin de saber el nivel de convivencia escolar de la muestra en estudio se tuvo los siguientes hallazgos de naturaleza descriptiva: de manera general, la convivencia escolar se encontró en un nivel medio y bajo de $30 \%$ y $42 \%$ respectivamente. En el diagnóstico general sobre el nivel de convivencia escolar de estudiantes, se pudo determinar que la mayor parte de estudiantes posee un mediano nivel de convivencia escolar; concluyendo que los estudiantes de dicha institución requieren una atención inmediata por tratarse de un tema central en su aprendizaje.

Se encontraron estudios referidos al tema de convivencia, como el de Trujillo, (2015), quien relaciona a la convivencia escolar con los valores, concluyendo que los valores influyen positivamente en la convivencia escolar. La teoría de los valores ha significado mucho a través de la historia y para las diversas sociedades ha tenido su significado propio; es por ello que aportan significativamente a la convivencia escolar. En concordancia con el anterior, Ramirez (2016) realizó un estudio muy grande en Madrid sobre convivencia escolar en instituciones secundarias, con una muestra de 22 y 571 estudiantes provenientes de varios países, cuyo objetivo fue demostrar la percepción de aquellos sobre la convivencia escolar. Para la presente investigación, respecto a la dimensión relaciones interpersonales, se logró determinar que, en su mayoría, los estudiantes de cuarto grado de nivel secundario de este complejo educativo se encuentran entre el nivel medio y bajo, siendo poco favorables a la Institución Educativa. Estos hallazgos son de manera virtual pues las relaciones humanas se encuentran como problemáticas que se debe mejorar.

Con relación al tema, Nicolas (2015) hace una investigación para determinar situación actual de la convivencia escolar en los centros educativos secundarios de la Región de Murcia. Los hallazgos fueron muy parecidos a los de Ramirez (2016) en países latinos, los estudiantes conciben positivamente la convivencia escolar; sin embargo, existen conflictos de diferente índole que hay que resolver. En el presente estudio, respecto a la dimensión disciplina democrática, se determinó que los estudiantes investigados poseían en su mayoría una carencia de esta, poniendo señales de alerta para atender como Institución Educativa.

Dentro de las investigaciones nacionales, la desarrollada por Cáceres (2017) tuvo como objetivo determinar la relación entre las habilidades sociales y la convivencia escolar de los estudiantes de 3 er año de secundaria. La conclusión a la que se llegó fue que existía una relación muy estrecha entre las habilidades sociales y la convivencia escolar en estudiantes de secundaria. En el presente estudio, respecto a la dimensión relación con el contexto se determinó que los estudiantes en cuestión tenían resultados desfavorables, ubicándose el mayor porcentaje en el nivel bajo. Una situación a todas luces preocupante, respecto a la cual es necesario tomar cartas en el asunto y asumir e compromiso institucional en la mejora de esta.

El estudio que más se acerca al nuestro es el de Barrientosetal.(2018)ensuinvestigación "Disposición al aprendizaje y convivencia democrática en escuelas públicas del Perú", el mismo que pretende identificar la relación entre la disposición al aprendizaje y la convivencia democrática en los estudiantes en los que se implementa el proyecto Comunidades de Aprendizaje, en regiones donde se aplica el proyecto: Lima, Callao, Piura, Cusco, Apurímac, Huancavelica y Puno, tanto en zonas urbanas y rurales. Los hallazgos fueron sorprendentes, alcanzando diferencias sustanciales en los resultados. Del mismo modo, respecto a la dimensión ciberconducta se obtuvo en el nivel medio a más de la mitad de encuestados y siendo que se está viviendo una educación de manera virtual y sumamente importante analizar y tomar acciones inmediatas para su mejora, es necesario que se atienda este aspecto.

Pero es así mismo importante resaltar algunas investigaciones de la variable independiente; en este caso las tertulias literarias. A nivel internacional, González (2016) hace un estudio en Madrid, cuyo objetivo principal fue identificar las características y comprobar las aportaciones que las Tertulias Pedagógicas Dialógicas pueden tener en el contexto de una formación continua; es decir, en la formación del profesorado. El más importante aporte de este estudio fue que se trabajaron con otros agentes de la comunidad educativa como padres y familiares de los docentes. Los resultados fueron muy favorables y beneficiosos ya que se trabajó con perfiles diversos superando barreras y dificultades que son propias de la escuela, notando un gran poder transformador. Concluyendo que trabajar con tertulias pedagógicas aportaba grandemente en los futuros profesores. Así mismo, Alonso et al. (2008) hacen un estudio en España, 
en donde proponen una estrategia que se centra en la utilización de las tertulias dialógicas como estrategia metodológica en la formación de adultos, obteniendo resultados favorables y mejorando asî la práctica educativa, pues el aprendizaje dialógico logró un gran impacto entre sus participantes. Finalmente, se concluye que es preciso aplicar una estrategia de tertulia literaria virtual que permita la mejora de la convivencia escolar en dicha institución por ser necesaria para los aprendizajes en un ambiente colaborativo.

De acuerdo con el estudio realizado se llegaron a las siguientes conclusiones:

1. Se caracterizó epistemológicamente la tertulia literaria virtual como herramienta para mejorar la convivencia escolar de la Institución Educativa Mallaritos al 2020, en donde la convivencia escolar se concibió como un conglomerado de relaciones humanas que toman lugar en una institución educativa, se forman de manera unificada entre todos sus miembros y todos ellos son responsables de la misma. Respetando en todo momento los derechos humanos, siendo consciente de igualdades y diferencias, basándose en el modelo humanista.

2. Se logró determinar el nivel de convivencia escolar en la I. E. Complejo Mallaritos, 2020, cuyos resultados se ubicaron en el nivel medio y bajo, resultados por ende desfavorables; lo que lleva a concluir que se necesita una estrategia para mejorar la convivencia escolar remota.

3. La aplicación de la tertulia literaria virtual como estrategia contribuirá al apoyo de la convivencia escolar en los estudiantes de cuarto grado de educación secundaria de la I. E. Complejo Mallaritos en el 2020, generando cambios significativos en el nivel de convivencia con sus pares.

4. Dar opciones a nuestros estudiantes para acceder a diferentes plataformas virtuales para poder llevar las tertulias literarias, asimismo, gestionar la conectividad como Institución Educativa. Promover una sana convivencia escolar virtual desde cualquier espacio en que se desarrollen las sesiones de aprendizaje, aplicando diversas estrategias para trabajar colaborativa y cooperativamente en beneficio de los aprendizajes.

\section{REFERENCIAS}

Alonso, M. J., Arandia, M. y Loza, M. (2008). La tertulia como estrategia metodológica en la formación continua avanzando en la dinámicas dialógicas. Revista Electrónica Interuniversitaria de Formación del Profesorado, 11(1), 1-7. file:///C:/Users/Javier/Downloads/
Dialnet-LaTertuliaComoEstrategiaMetodologicaEnLaFormacionC-2783507.pdf

Álvarez, C., Cotado, L. y Iturriaga, A. (2012, 27, 28 y 29 de junio). Aprendizaje dialógico, grupos interactivos y tertulias literarias: una apuesta de centro educativo que favorece la inclusión. Estilos de Aprendizaje: Investigaciones y Experiencias : [Ponencia] V Congreso Mundial de Estilos de Aprendizaje, Santander, España. https://dialnet.unirioja.es/servlet/articulo?codigo $=4644436$

Andina, Agencia peruana de noticias. (2019). Minedu: cerca de 12,000 casos de violencia escolar se reportaron este año. Andina https://www.andina.pe/agencia/ noticia-minedu-cerca-12000-casos-violencia-escolar-se-reportaron-este-ano-779342.aspx

Arias, J., Villasís, A., Keever, M., Guadalupe, M. y Novales, M. (2016). Metodología de la investigación. Alergia México, 63, 201-206. www.nietoeditores.com.mx

Arrunátegui, G. (2020). Una aproximación a la violencia en escuelas primarias muligrado de zonas rurales de Piura, Cajamarca y Loreto [Archivo PDF]. GRADE. http:// repositorio.grade.org.pe/handle/GRADE/

Barrientos, R., Yamanija, C. y Omura, D. (2018). Disposición a aprendizaje y convivencia democrática en escuelas públicas del Perú. Revista EDUCA UMCH, 11, 51-84. https://doi.org/10.35756/educaumch.v11i0.66

Cáceres, R. (2017). Habilidades sociales y conducta agresiva en estudiantes del 2do año de secundaria de la I.E "Santa Rosa" - Tarapoto [Tesis de maestría, Universidad César Vallejo]. Repositorio de la Universidad César Vallejo. http://repositorio.ucv.edu.pe/handle/20.500.12692/5855

Carozzo, J. (2016). Dimensiones de la convivencia. Huellas, 3(5), 27-42. http://www2.congreso.gob. pe/sicr/cendocbib/con4_uibd.nsf/DC5D2EB382546263052580A600733653/\$FILE/Dimensiones.pdf

Casas, J., Repullo, J. R. y Donado, J. (2003). La encuesta como tecnica de investigacion. Atención Primaria, 31 (8), 527-538. http://www.unidaddocentemfyclaspalmas. org.es/resources/9+Aten+Primaria+2003.+La+Encuesta+l.+Custionario+y+Estadistica.pdf

Redacción EC. (2019). Aumentan casos de denuncias de ciberacoso por facebook y whastapp. El Comercio. https://elcomercio.pe/videos/pais/peru-aumentan-casos-de-denuncia-de-ciberacoso-por-redes-sociales-nnav-noticia/

González, V. (2016). De la formación permanente del profesorado a la formación dialógica de la comunidad: análisis de una tertulia pedagógica comunitaria http://eprints.ucm.es/40521/1/T38145.pdf

Hernández, R., Fernandez, C. y Baptista, P. (2014). Metodología de la investigación ( $6^{a}$ ed.). McGraw-Hill. http:// observatorio.epacartagena.gov.co/wp-content/ uploads/2017/08/metodologia-de-la-investigacion-sexta-edicion.compressed.pdf

Ministerio de Educación - Ministerio de Educación-MINEDU. (2016). Evaluación Censal de Estudiantes 2015 
[Archivo PDF]. MINEDU. http://umc.minedu.gob.pe/ wp-content/uploads/2016/03/Informe-para-el-docente-Lectura_ECE-2015.pdf

Ministerio de Educación - Ministerio de Educación-MINEDU. (2017). Participación y clima institucional para una organización escolar efectiva. Texto del módulo 3 [Archivo PDF]. MINEDU. https://repositorio.minedu. gob.pe/handle/20.500.12799/5922.

Ministerio de Educación-MINEDU. (2018). Escolar, La Prevención Y La Atención De La. 56. http://repositorio. minedu.gob.pe/bitstream/handle/MINEDU/6088/ Lineamientos para la gestión de la convivencia escolar\%2C la prevención y la atención de la violencia contra niñas\%2C niños y adolescentes.pdf?sequen$\mathrm{ce}=1 \&$ is Allowed $=\mathrm{y}$

Ministerio de Educación - Ministerio de Educación-MINEDU (2020). Orientaciones generales para la prevención de riesgos virtuales [Archivo PDF]. MINEDU. http:// www.ugelcp.gob.pe/documentos_agp/seguridad-04-estudiante-orientaciones-generales-estudiantes.pdf

Morales, M., \& López, V. (2019). Políticas de convivencia escolar en América Latina: Cuatro perspectivas de comprensión y acción. Archivos Analíticos de Políticas Educativas, 27(5), 1-28. https://doi.org/10.14507/ epaa. 27.3800

Nicolas, A. (2015). La convivencia escolar en los centros de Educación Secundaria de la Región de Murcia: La voz del alumnado. [Tesis de Doctorado] Universidad de Murcia. https://dialnet.unirioja.es/servlet/tesis?codigo $=128396$

Ramirez, J. J. (2016). Convivencia escolar en instituciones de educación secundaria: un estudio transcultural [Tesis doctoral, Universidad Complutense de Madrid]. E-Prints Complutense. https://eprints.ucm. es/40428/
Rodríguez, M. y Mendivelso, F. (2018). Diseño de investigación de Corte Transversal. Revista Médica Sanitas, 21(3), 141-146. https://doi.org/10.26852/01234250.20

Trujillo, D. (2015). Convivencia escolar y valores en estudiantes de grado octavo y noveno de la Institución Educativa Policarpa Salavarrieta del municipio de Quimbaya (Quindío). [Tesis doctoral, Universidad Norbert Wiener]. DSpace Repository. http://repositorio.uwiener. edu.pe/handle/123456789/1730

UNESCO. (2019). Behind the numbers: Ending school violence and bullying. Organización de las Naciones Unidas para la Educación, la Ciencia y la Cultura-UNESCO.

Vargas, J. y Ramón, F. (2016). ¿Cómo se resuelven los conflictos en tres comunidades de aprendizaje? Revista de Psicodidactica, 21(2), 281-301.

Yuni, J. y Urbano, C. (2014). Técnicas para investigar. Recursos Metodológicos para la Preparación

de Proyectos de Investigación. Brujas. https://abacoenred. com/wp-content/uploads/2019/01/LIBRO-T\%C3\%A9cnicas-para-investigar-1.pdf

\section{Contribución de los autores}

GCM-U: resumen, introducción, métodos y discusión. GC-B: resultados y revisión final del artículo.

Fuentes de financiamiento

La investigación fue realizada con recursos propios.

Conflictos de interés

Los autores declaran no tener conflicto de interés.

\section{Correspondencia}

Gely Coraly Martinez-Ubillús

Dirección: Calle José Balta 121, Sullana, Piura, Perú.

Cel.: +51969992690

Email: gmartinezub12@ucvvirtual.edu.pe 